\title{
Commentary \\ More than skin deep: moisturizing body milk and Burkholderia cepacia
}

\author{
Amy E Irwin ${ }^{1}$ and Connie Savor Price ${ }^{1,2}$
}

1Division of Infectious Diseases, Denver Health and Hospital Authority, 777 Bannock Street, Denver, CO, 80238 USA

${ }^{2}$ University of Colorado School of Medicine, Campus Box C290, 4200 E. Ninth Ave., Denver, CO 80262 USA

Corresponding author: Connie S Price, connie.price@dhha.org

Published: 20 February 2008

Critical Care 2008, 12:115 (doi:10.1186/cc6781)

This article is online at http://ccforum.com/content/12/1/115

(C) 2008 BioMed Central Ltd

See related research by Alvarez-Lerma et al., http://ccforum.com/content/12/1/R10

\begin{abstract}
Alvarez-Lerma and colleagues observed over an 18-day period that five critically ill patients admitted to a multidisciplinary 18-bed intensive care unit contracted Burkholderia cepacia from unopened containers of moisturizing body milk, calling into question the use in critical care settings of cosmetic products that do not guarantee sterilization during the manufacturing process. Is this the answer to the problem, however, or should the use of lotions in such settings be re-examined?
\end{abstract}

It appears that the potentially fatal pathogen Burkholderia cepacia has taken hold of another reservoir for transmission in the form of moisturizing body milk [1]. B. cepacia, a Gramnegative rod bacterium known for its affinity towards and its virulence in moist environments, presents a clinical challenge in the treatment and management of susceptible populations and in the subsequent identification of causative sources during nosocomial outbreaks. Capable of person-to-person transmission and transmission through contact with surfaces such as medical devices and medicines, $B$. cepacia is an opportunistic pathogen highly resistant to most antimicrobial agents and it possesses a $42 \%$ mortality rate [2]. The need for all clinicians to examine more closely their environments in an effort to halt the spread of $B$. cepacia is therefore critical.

Alvarez-Lerma and colleagues observed over an 18-day period that five critically ill patients admitted to a multidisciplinary 18-bed intensive care unit contracted the nosocomial infection B. cepacia [1]. Microbiologic analysis was performed on the collected oropharyngeal mucosa, urine, and bronchial aspirates from all patients in the intensive care unit and on samples of antiseptics, eau de Cologne, and moisturizing body milk used in routine nursing care. It was determined that the strains of $B$. cepacia isolated from the patients and from the moisturizing body milk were of the same clone, indicating the lotion was the source of the out- break. Upon this discovery, the hospital immediately withdrew the product from routine nursing care hospital wide and notified the manufacturing company about the $B$. cepacia contamination.

Alvarez-Lerma and colleagues strongly recommend cosmetic products that do not guarantee sterilization during the manufacturing process should not be used in critical care settings [1]. From an all or nothing perspective and within the context that $B$. cepacia only survived in moisturizing body milk, this approach would appear practical and the right thing to do in an effort to promote quality and safe patient care. Removing nonsterile cosmetic products such as lotion from the critical care setting, however, would not completely eradicate or protect patients from $B$. cepacia infecting humans in the hospital setting. The fact remains that $B$. cepacia has the potential to find its way into reservoirs that have undergone sterile manufacture because of its affinity for moist environments. Several recent studies illustrate that strains of the $B$. cepacia complex were found in antiseptics, ultrasound gel, enteral feedings, and albuterol and nasal sprays - products manufactured in sterile environments [3-6].

In an effort to first do no harm, the clinician must carefully weigh the risks and benefits of all interventions for every patient. The critically ill patient in a state of imbalance is more susceptible and vulnerable to pathogens in their environment; therefore, a greater need for enhanced analysis of the benefits and risks inherently exists. It is feasible to ban the use of lotions in critically ill patients if it is known that $B$. cepacia clinically demonstrates higher colonization rates within moisturizing body milks than any other products, sterilized or nonsterilized, used in the hospital. Without such evidence that lotions possess a higher colonization rate for $B$. cepacia, from a nursing perspective the potential benefits of the use of nonsterilized lotion for the patient might be overlooked. 
Nursing practice utilizes lotion in a variety of situations and patient populations. Lotion, commonly applied to patients with dry, itchy skin, helps to decrease scratching, which can lead to skin excoriation and can leave the body open to infection [7]. Lotion may also be utilized in patients who are bed-ridden and who present with red areas on bony prominences. Here, the lotion helps to create a gentle friction on the reddened areas of the body, which helps to stimulate blood flow to the surrounding tissue in an effort to ward off potential development of pressure ulcers. Additionally, the use of lotions can assist in the donning of latex-free compression stockings commonly used in a multitude of medical conditions such as lymphedema and venous insufficiency. From a holistic perspective, lotions are used to facilitate patient massage. The use of aids in the bonding process of parents of babies in the neonatal intensive care unit by helping to facilitate infant massages, and helps facilitate sensory integration therapies in children with autism and sensory processing disorders.

There is no doubt of the tragic magnitude that would ensue if a widespread deadly outbreak of $B$. cepacia was to occur from the use of moisturizing body lotions, especially if those greatly affected were infants, children, and adults concurrently battling critical or chronic illnesses. Halting the spread of $B$. cepacia infection cannot occur only through the discontinuation of nonsterilized cosmetic products, and neither can it be guaranteed only with the use of sterilized products. A tried and true way to take hold of $B$. cepacia therefore appears to be through the continued and regimented practice of adequate hand washing.

\section{Competing interests}

The authors declare that they have no competing interests.

\section{References}

1. Alvarez-Lerma F, Marull E, Terradas R, Segura C, Planells I, Coll P, Knobel $\mathrm{H}$, Vazquez $A$ : Moisturizing body milk as a reservoir of Burkholderia cepacia: outbreak of nosocomial infection in a multidisciplinary intensive care unit. Crit Care 2008, 12:R10.

2. Shehabi A, Abu-al-soud W, Mahafsah A, Khuri-bulos N, Ouis I, Watrom T: Investigation of burkholderia cepacia nosocomial outbreak with high fatality in patients suffering from diseases other than cystic fibrosis. Scand J Infect Dis 2004, 36:174-178.

3. Godshall M: Mounting a defense against Burkholderia cepacia. Nursing 2005, 35:18-19.

4. Jacobson M, Wray R, Kovach D, Henry D, Speert D, Matlow A: Sustained endemicity of Burkholderia cepacia complex in a pediatric institution, associated with contaminated ultrasound gel. Infect Control Hosp Epidemiol 2006, 27:362-398.

5. Nasser R, Rahi Am, Haddad M, Daoud Z, Irani-Hakime N, Almawi W: Outbreak of Burholderia cepacia bacteremia traced to contaminated hospital water used for dilution of an alcohol skin antiseptic. Infect Control Hosp Epidemiol 2004, 25:231239.

6. Estivariz $C$, Bhatti $L$, Pati $R$, Jensen $B$, Arduino M, Jernigan D, LiPuma J, Srinivasan A: An outbreak of Burholderia cepacia associated with contamination of albuterol and nasal spray. Chest 2006, 130:1346-1353.

7. Schreiner $\mathrm{T}:$ Oh, the weather outside is frightful ... apply basic skin care and moisturizers to relieve dry, chapped winter skin. Adv Nurs 2007, 9:19-20. 